\title{
Co-delivery of doxorubicin and oleanolic acid by triple-sensitive nanocomposite based on chitosan for effective promoting tumor apoptosis
}

Xia Chen

Shiwei Niu

David H. Bremner

Xuejing Zhang

Hongmei Zhang

Yanyan Zhang

Shude Li

Li-Min Zhu

This is the accepted manuscript (C 2020, Elsevier Licensed under the Creative Commons AttributionNonCommercial-NoDerivatives 4.0 International (CC BYNC-ND 4.0)

\section{(Cc) BY-NC-ND}

The published article is available from doi: http://dx.doi.org/10.1016/j.carbpol.2020.116672 


\section{Co-delivery of doxorubicin and oleanolic acid by}

2 triple-sensitive nanocomposite based on chitosan for

\section{3 effective promoting tumor apoptosis}

4 Xia Chen ${ }^{\mathrm{a} \dagger}$, Shiwei Niu ${ }^{\mathrm{ab} \dagger}$, David H. Bremner ${ }^{\mathrm{c}}$, Xuejing Zhang ${ }^{\mathrm{a}}$, Hongmei Zhang ${ }^{\mathrm{a}}$, Yanyan Zhang ${ }^{\mathrm{a}}$,

$5 \quad$ Shude $\mathrm{Li}^{\mathrm{d}^{*}}, \mathrm{Li}-\mathrm{Min} \mathrm{Zhu}^{\mathrm{a}^{*}}$

$6 \quad{ }^{a}$ College of Chemistry, Chemical Engineering and Biotechnology, Donghua University, Shanghai,

$7 \quad$ PR China

$8 \quad$ bcience and Technology Achievement Incubation Center, Kunming Medical University, Kunming

9 650500, PR China

10 'School of Science, Engineering and Technology, Kydd Building, Abertay University, Dundee DD1

11 1HG, Scotland, UK

$12{ }^{d}$ Department of Biochemistry and Molecular Biology, School of Basic Medicine, Kunming Medical

13 University, Kunming 650500, PR China

14

$15+$ Co-first Authors, contributed equally to this work.

16 *Authors for correspondence: Li-Min Zhu.Tel: 008621 67792655.Email: lzhu@dhu.edu.cn;

17 Shude Li.Tel: 13529227380.Email: shudeli006@vip.sina.com. 


\section{ABSTRACT}

Nanocomposites as "stevedores" for co-delivery of multidrugs hold great promise in addressing the drawbacks of traditional cancer chemotherapy. In this work, our strategy presents a new avenue for the stepwise release of two co-delivered agents into the tumor cells. The hybrid nanocomposite consists of a pH-responsive chitosan (CS), a thermosensitive poly( $N$-vinylcaprolactam) (PNVCL) and a functionalized cell-penetrating peptide (H6R6). Doxorubicin (DOX) and oleanolic acid (OA) are loaded into the nanocomposite (H6R6-CS-g-PNVCL). The system displayed a suitable size $(\sim 190 \mathrm{~nm})$, a high DOX loading $(13.2 \%)$ and $\mathrm{OA}$ loading efficiency $(7.3 \%)$. The tumor microenvironment triggered the nanocomposite to be selectively retained in tumor cells, then releasing the drugs. Both in vitro and in vivo studies showed a significant enhancement in antitumor activity of the co-delivered system in comparison to mono-delivery. This approach which relies on redox, $\mathrm{pH}$ and temperature effects utilizing co-delivery nanosystems may be beneficial for future applications in cancer chemotherapy.

Keywords: chitosan; H6R6 peptide; triple sensitivity; controlled release; chemotherapy

\section{Introduction}

To date, cancer remains one of the most threatening intractable diseases in human medical history and although there are numerous treatments, chemotherapy still plays an irreplaceable role in treatment (X. Chen et al., 2019; J. Wang et al., 2019). However, conventional chemotherapy exhibits many drawbacks such as poor water-solubility of the anticancer drugs, damaging side-effects on normal cells, the premature leakage of drugs from delivery systems, as well as the insignificant therapeutic effect when only a single drug is utilized. Furthermore, long-term administration of chemotherapeutics can cause multidrug resistance (MDR) of tumor cells, which results in the failure of chemotherapy (Chen et al., 2016; Zhao et al., 2017; Zhou et al., 2018). Fortunately, various nanotechnologies have been introduced into drug delivery systems to solve some of these problems (Chai et al., 2019; Jin et al., 2019; Ke et al., 2019; Lang et al., 2019). The enhanced permeability and retention (EPR) effect of solid tumors can make it easier for nanocomposites of suitable size to accumulate in tumor tissues (Luo et al., 2019; Y. Zhang, Lu, et al., 2017), thereby facilitating the effective delivery of chemotherapy drugs. In addition, tumor 
cells possess their own peculiarities that provide many opportunities for cancer treatment (El-Sawy, Al-Abd, Ahmed, El-Say, \& Torchilin, 2018), including an acidic microenvironment (J. Xiao et al., 2019; L. Xiao, Huang, Moingeon, Gauthier, \& Yang, 2017; Y. Zhang, Dang, et al., 2017), higher temperature within cancer cells (S. Niu et al., 2018) and greater intracellular glutathione (GSH) concentration than that observed in normal cells (Hu et al., 2016; Yang et al., 2019). In recent years, specific tumor microenvironments have led to the design of an increasing number of nanodrug delivery systems to treat cancer. For example, Wang's group reported a $\mathrm{pH}$ and reduction-responsive polymeric lipid vesicle for the targeted delivery of doxorubicin (S. Wang et al., 2014). Mao's group designed a micelle to control the release of doxorubicin under a high GSH tumor microenvironment (Mao et al., 2018). The use of such specialized treatments utilizing these particular tumor microenvironments have paved the way for novel targeted cancer chemotherapy.

According to previous literature reports, chitosan (CS) has a variety of beneficial properties in vivo e.g. amphipathy, biocompatibility, biodegradability and biosafety (Galbiati et al., 2011). CS can inhibit bacterial infection, decrease inflammation (L. Sun et al., 2019), and is soluble in acidic solutions (Rosiere et al., 2018), thereby allowing it to respond to the slightly acidic microenvironment found in tumors. There are also many applications of CS in the field of drug delivery, for example, Niu's group developed a chitosan-based cascade-responsive doxorubicin delivery system to overcome some hard-to-treat cancers (Shiwei Niu, Williams, Wu, Wu, Zhang, Chen, et al., 2019) and Zhang's group synthesized hyaluronic acid-grafted chitosan nanoparticles to improve delivery of paclitaxel for breast cancer therapy (X. Zhang et al., 2019). Poly( $N$-vinylcaprolactam) (PNVCL), a promising thermo-responsive polymer, shows a lower critical solution temperature (LCST) at $\sim 32^{\circ} \mathrm{C}$ (Montes, Ortega, \& Burillo, 2014) and the LCST can be tuned to a higher temperature by grafting on other hydrophilic polymers (Indulekha, Arunkumar, Bahadur, \& Srivastava, 2017). Therefore, PNVCL has been widely applied in drug delivery and thermo-triggered drug release systems (Prabaharan, Grailer, Steeber, \& Gong, 2009). Several teams have taken advantage of the combination of CS and PNVCL for cancer theranostics (Shiwei Niu, Williams, Wu, Wu, Zhang, Chen, et al., 2019). Active targeting of drug delivery systems can be enhanced by the use of cell-penetrating peptides (CPPs) which are well known short peptides that can assist in penetration of certain substances into cells through endocytosis 
(Xu et al., 2019). There are various reviews of therapeutic applications of CPPs in different tissues and organs (Gao et al., 2019; Hingorani et al., 2020; Liu et al., 2019; Wu et al., 2019). According to a recent report (P. Sun et al., 2017), the H6R6 peptide (amino acid sequence: KVRVRVRVDPTRVRERVK) is one of the CPPs showing remarkable mediating effects, which can facilitate the uptake of drug-loaded nanoparticles (NPs) by tumor cells. An important factor in the mediation of endocytosis is the presence of an appropriate quantity of arginine amino acids in H6R6 peptide which renders it positively charged and as cell membranes are negatively charged the electrostatic interaction facilitates endocytosis (Perche, 2019). Furthermore, the H6R6 peptide increases intracellular uptake and helps NPs escape from the endosome/lysosome (P. Sun et al., 2017), which is advantageous for drugs diffusing into the cytoplasm to kill the tumors.

On the other hand, cancer can also be treated with multiple chemotherapy drugs. In addition to doxorubicin (DOX), oleanolic acid (OA) is an excellent naturally occurring anticancer agent with hepato-protective action (Tao et al., 2018). Recent studies have shown that OA can inhibit the growth of leukemia, non-small-cell lung cancer (NSCLC) cell lines (Z. Chen et al., 2019) and other malignant tumors (Silva et al., 2019; Takemura et al., 2011). Furthermore, it has been shown that OA can address MDR (Shiwei Niu, Williams, Wu, Wu, Zhang, Zheng, et al., 2019), so it may be highly beneficial to load nanocomposites with two drugs (DOX and OA) for broad-spectrum growth inhibition of tumors.

In this current work, the nanocomposite was fabricated by reversible addition fragmentation chain transfer (RAFT) polymerization to form the thermo- and $\mathrm{pH}$ - sensitive copolymer (CS-g-PNVCL). The H6R6 peptide was bound to the CS-g-PNVCL by reduction-sensitive disulfide bonds (-S-S-) to form H6R6-CS-g-PNVCL (self-assembly in aqueous solution) which had the hydrophilic H6R6 peptide on the outermost surface of the nanocomposite. Finally, the hydrophobic chemotherapeutics (DOX and OA) were physically loaded into the hydrophobic core of the nanocomposite to obtain the drug co-delivery system (DOX/OA)@H6R6-CS-g-PNVCL. Therefore, the role of CS is to promote the disintegration of the nanocomposite in the acidic tumor microenvironment, the role of NVCL is to make the LCST of the nanocomposite be tuned to a higher temperature (close to the temperature of the tumor microenvironment), promoting the further disintegration of the nanocomposite in the temperature of the tumor microenvironment. Once the NPs reach the tumor cells, the outermost H6R6 peptide will detach from the NPs via 
breakage of -S-S- bonds under the reductive tumor microenvironment. The combination of -S-Sbonds and the H6R6 peptide plays an important role in allowing the nanocomposite to stay in the tumor cells. Thereafter, the relatively high temperature and acidic conditions of the tumor further promote the disintegration of the hybrid nanocomposite, triggering release of the drugs. And the design of such nanocomposites and the strategy of dual-loaded drugs may provide a new direction in tumor chemotherapy.

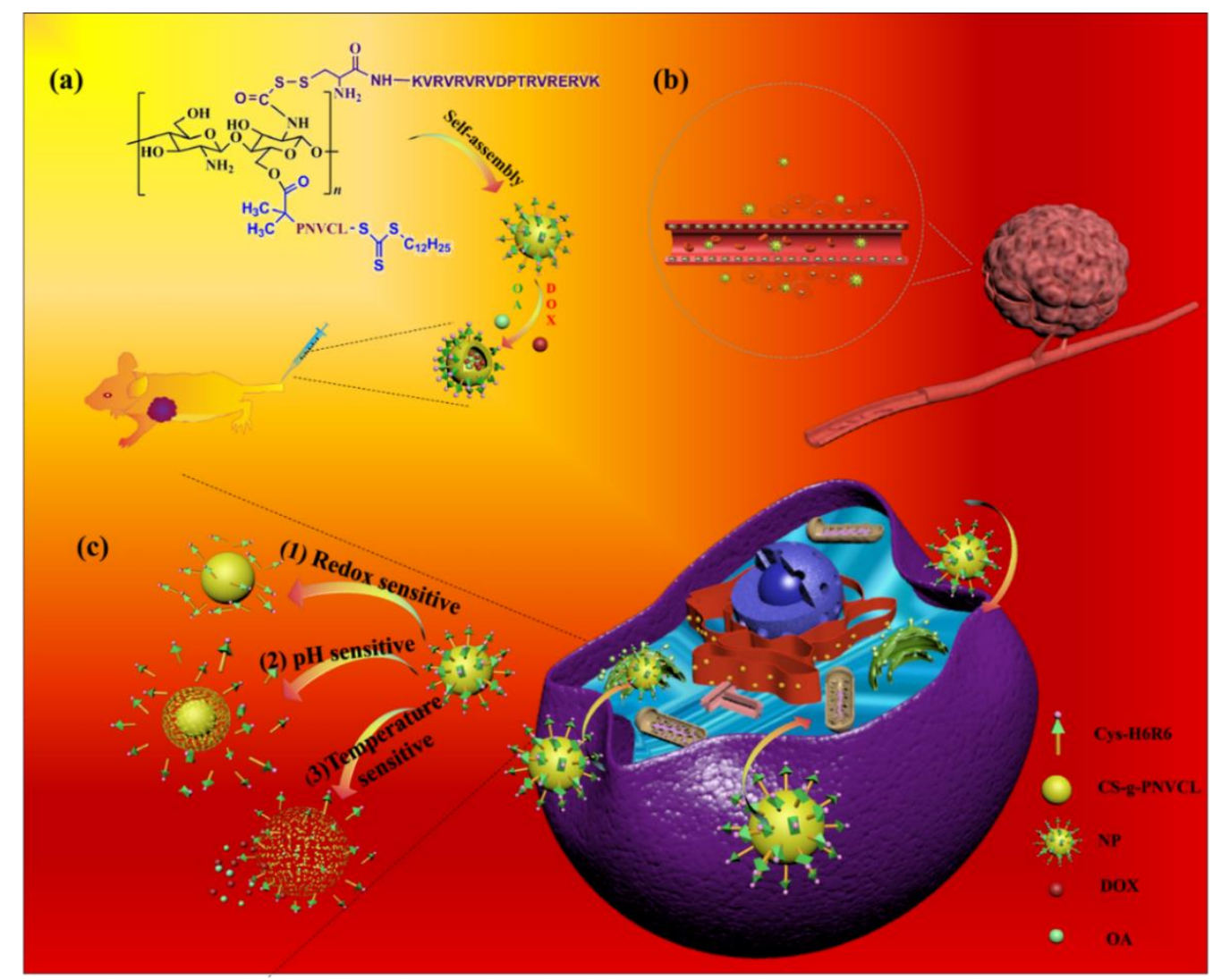

Scheme 1. (a) The self-assembly of H6R6-CS-g-PNVCL NPs. (b) The enhanced penetration and retention effect ensures that the NPs remain in the tumor sites. (c) The self-destructive behavior of NPs with triple sensitivity in the tumor microenvironment, releasing DOX and OA.

\section{Experimental details}

\subsection{Materials, cell lines and animals}

Chitosan (CS, degree of deacetylation $>95 \%$, Mw $200 \mathrm{kDa}$ ), $N, N$-dimethylformamide (DMF) and acetic anhydride were sourced from the SinoPharm Chemical Reagent Co., Ltd (Shanghai, China). $\quad N$-Vinylcaprolactam (NVCL) and dialysis tubing were supplied by the Titan Technology 

purchased from DG Peptides Co., Ltd. (Hangzhou, China). Doxorubicin (DOX, 98\%), $N$-(3-dimethyl-aminopropyl)- $N$-ethylcarbodiimide hydrochloride (EDC), azobisisobutyronitrile (AIBN), $S^{\prime}-1$-dodecyl- $S^{\prime}-\left(\alpha, \alpha^{\prime}\right.$-dimethyl- $\alpha^{\prime}$-acetic acid) trithiocarbonate (DDATC) and $N$-hydroxysuccinimide (NHS) were obtained from the Aladdin Reagent Co. (Shanghai, China). Deuterium oxide $\left(\mathrm{D}_{2} \mathrm{O}\right)$, Dimethyl sulfoxide-d6 (DMSO-d6), 4-(dimethyl-amino) pyridine (DMAP), $N, N$-dicyclohexylcarbodiimide (DCC), thioglycolic acid, glutathione (GSH) and oleanolic acid (OA) were procured from Sigma-Aldrich (St. Louis, MO, USA). Dulbecco's modified Eagle medium (DMEM), 3-(4,5-dimethylthiazol-2-yl)-2,5-diphenyltetrazolium bromide (MTT), fetal bovine serum (FBS), McCoy's 5A incomplete medium, 0.05\% trypsin-EDTA, $0.25 \%$ trypsin (without EDTA), Hoechst 33342, calcein-AM, propidium iodide (PI), Annexin V-FITC/PI cell apoptosis analysis kit and phosphate buffered solution (PBS) were obtained from the Beyotime Institute of Biotechnology (Shanghai, China). DiR (1,10-dioctadecyl-3,3,30,30-tetramethylindo- tricarbo cyanine iodide) was sourced from Biotium (Fremont, CA, USA). All chemicals were of analytical grade and had no additional purification.

\subsection{Synthesis of H6R6-CS-g-PNVCL}

H6R6-CS-g-PNVCL was synthesized using the literature method with some modifications (Qian et al., 2019; Quinones, Peniche, \& Peniche, 2018). The procedure was that we introduced the hydrophobic NVCL into CS molecules by RAFT reagent grafting to generate a hydrophobic-hydrophilic balance promoting the copolymer (CS-g-PNVCL) self-assembly, then hydrophilic H6R6 peptide was modified on the outermost surface of the copolymer. Chitosan (CS, 
$5 \mathrm{~g})$ was fully dissolved in aqueous acetic acid $(250 \mathrm{~mL}, 1.0 \% \mathrm{w} / \mathrm{w})$ and stirred with anhydrous ethanol $(250 \mathrm{~mL})$ until clarified, acetic anhydride $(0.5 \mathrm{~mL})$ was added and stirring continued at room temperature for $12 \mathrm{~h}$. An aqueous solution of $\mathrm{NaOH}(50 \mathrm{~mL}, 10 \% \mathrm{w} / \mathrm{w})$ was added and the precipitate was removed by filtration and the resulting solution was lyophilized to obtain $N$-acetyl CS. The $N$-CS-RAFT was prepared by dissolving $N$-acetyl CS $(0.292 \mathrm{~g})$ in DMF $(30 \mathrm{~mL})$ and stirring with DDACT $(0.37 \mathrm{~g}), \mathrm{DCC}(0.205 \mathrm{~g})$ and DMAP $(0.015 \mathrm{~g})$ at room temperature for $40 \mathrm{~h}$. Ultrapure ice water was added and the precipitate was dialyzed with a dialysis tubing (molecular weight cutoff $(\mathrm{MWCO})=3500 \mathrm{Da})$ for $3 \mathrm{~d}$, followed by freeze-drying for $3 \mathrm{~d}$, to produce the golden yellow $N$-acetyl CS-RAFT.

The $N$-acetyl CS-RAFT (0.0468 g) was then dissolved in DMF (5 mL) and AIBN ( $0.0032 \mathrm{~g})$ and NVCL $(1 \mathrm{~g})$ were added and gently stirred in an oil bath at $60{ }^{\circ} \mathrm{C}$ under nitrogen until the reaction mixture was completely dissolved (about $4 \mathrm{~h})$. Ice-cold diethyl ether $(50 \mathrm{~mL})$ was poured into the reaction mixture about $12 \mathrm{~h}$ later to form a precipitate which was collected by filtration. The precipitate was then dialyzed $(\mathrm{MWCO}=3500 \mathrm{Da})$ for $3 \mathrm{~d}$ to remove any unreacted starting materials and then the dialyzed solution was freeze-dried to afford $N$-acetyl CS-g-PNVCL. Finally, the $\mathrm{N}$-acetyl groups were removed by hydrolysis for $24 \mathrm{~h}$ with aqueous $\mathrm{NaOH}\left(0.01 \mathrm{~g} \mathrm{NaOH}\right.$ in $\left.0.09 \mathrm{~mL} \mathrm{H}_{2} \mathrm{O}, \mathrm{pH}=10\right)$ and the CS-g-PNVCL was collected by dialysis and drying.

Finally, H6R6-CS-g-PNVCL was prepared by dissolving CS-g-PNVCL (0.05 g) in PBS buffer solution (30 mL, pH 7.4), reacting it with thioglycolic acid (20 mg) catalysed with EDC (20 $\mathrm{mg})$ and NHS (6.4 mg) and stirring under nitrogen at room temperature for $2 \mathrm{~h}$. Cysteine-modified H6R6 peptide (0.04 g) was dissolved in PBS buffer solution (10 mL, pH 7.4) and slowly added dropwise into the above solution with stirring at room temperature in the dark under nitrogen for $24 \mathrm{~h}$. The solution were dialyzed $(\mathrm{MWCO}=3500 \mathrm{Da})$ for $3 \mathrm{~d}$, and freeze-dried to obtain H6R6-CS-g-PNVCL.

\subsection{Drug loading and characterization}

The hydrophobic chemotherapeutics (DOX and OA) were physically loaded into the hydrophobic core of the nanocomposite. DOX (5 mg) and OA (5 mg) were dissolved in DMF (10 $\mathrm{mL})$ and H6R6-CS-g-PNVCL (30 mg) was dissolved in PBS solution (25 mL, $\mathrm{pH} 7.4)$ and the two 
solutions were mixed and dispersed with ultrasonic assistance followed by stirring in the dark for $24 \mathrm{~h}$. The resulting product was centrifuged $(3,000 \mathrm{r} / \mathrm{min})$ for $15 \mathrm{~min}$ and dialyzed $(\mathrm{MWCO}=$ $3500 \mathrm{Da}$ ) for $3 \mathrm{~d}$, then freeze-dried to obtain (DOX/OA)@H6R6-CS-g-PNVCL as a powder. The preparation of DOX@H6R6-CS-g-PNVCL was similar to that of (DOX/OA)@H6R6-CS-g-PNVCL except OA was added. (DOX/OA)@CS-g-PNVCL was prepared without adding any cysteine-modified H6R6 peptide.

A small amount of (DOX/OA)@H6R6-CS-g-PNVCL powder was dissolved in ultra-pure water and then centrifuged $(11,000 \mathrm{r} / \mathrm{min})$ for $1 \mathrm{~h}$. The supernatant was collected and the amount of unencapsulated drugs were determined by UV-vis spectrophotometry (UNICO, China) using previously prepared calibration curves (Fig. S5). The encapsulation efficiency (EE) and drug loading (DL) of DOX were calculated using equation (1) and (2), respectively, and the encapsulation efficiency (EE') and drug loading (DL') of OA were calculated from equation (3) and (4), respectively.

$\mathrm{DOX}_{\mathrm{t}}$ is the total mass $(\mathrm{mg})$ of DOX to fabricate the nanocomposite; $\mathrm{DOX}_{\mathrm{f}}$ is the mass $(\mathrm{mg})$ of unencapsulated DOX in the supernatant; $\mathrm{G}$ is the total mass $(\mathrm{mg})$ of the nanocomposite; $\mathrm{OA}_{\mathrm{t}}$ is the total mass $(\mathrm{mg})$ of $\mathrm{OA}$ used to fabricate the nanocomposite; and $\mathrm{OA}_{\mathrm{f}}$ is the mass $(\mathrm{mg})$ of unencapsulated OA in the supernatant.

Fourier transform infrared (FT-IR) spectra were recorded using a Nicolet-Nexus 670 spectrometer (Thermo Fisher, USA.) over the range $4000-500 \mathrm{~cm}^{-1}$ with the resolution of $2 \mathrm{~cm}^{-1}$. To further confirm the successful preparation of NPs, ${ }^{1} \mathrm{H}$ NMR spectra and ${ }^{13} \mathrm{C}$ NMR spectra were obtained on a Bruker AVANCE $400 \mathrm{M}$ spectrometer (Bruker, USA.) with $\mathrm{D}_{2} \mathrm{O}$ or DMSO-d6 as the solvent. The size and morphology of (DOX/OA)@H6R6-CS-g-PNVCL were visualized using a transmission electron microscope (JEM 1200EX instrument, JOEL, Japan) and a scanning electron microscope (Nova TM Nano instrument, FEI, USA.). The size distribution and zeta 
potential of NPs were determined by dynamic light scattering (DLS) using a BI-200SM instrument (Brookhaven Instruments, USA.). The thermogravimetric analysis (TGA) of the samples from $100{ }^{\circ} \mathrm{C}$ to $800{ }^{\circ} \mathrm{C}$ were recorded using a thermogravimetric analyzer (Netzsch TG 209 F1 Libra, Germany). The LCST of the nanocomposites was recorded with a UV-vis spectrophotometer (PerkinElmer, Waltham, MA, USA), and the concentration of the sample dispersion is $0.5 \mathrm{mg} / \mathrm{mL}$ ( $\mathrm{pH} 7.4)$.

\subsection{In vitro $D O X$ and $O A$ release}

The dialysis method was used to study the release behaviors of (DOX/OA)@H6R6-CS-g-PNVCL at different temperatures and pH (mimics normal cell and specific tumor microenvironments). In order to explore the effect of $\mathrm{pH}$ on in vitro release, (DOX/OA)@H6R6-CS-g-PNVCL powders (5 mg) were fully dissolved in PBS buffer solution (5 $\mathrm{mL}, \mathrm{pH} 7.4)$ and PBS buffer solution (5 $\mathrm{mL}, \mathrm{pH} 6.5)$, respectively and the above two solutions were poured into dialysis tubing $(\mathrm{MWCO}=3500 \mathrm{Da})$, placed in the containers containing the same PBS solution $(100 \mathrm{~mL})$ and shaken at a frequency of 100 oscillations $/ \mathrm{min}$ in a $37{ }^{\circ} \mathrm{C}$ water bath. Samples of PBS solution $(1 \mathrm{~mL})$ were removed from the container at certain time intervals and supplemented with the same preheated PBS buffer solution $(1 \mathrm{~mL})$. The amount of DOX or OA in the samples was determined by UV-vis spectrophotometry. The above experiments were repeated but the temperature was changed to $40{ }^{\circ} \mathrm{C}$ in order to explore the effect of temperature on in vitro drug release. Each experiment was repeated three times.

\subsection{Cell culture}

SKOV3 cells were cultured in McCoy's 5A incomplete medium (containing $80 \mathrm{U} / \mathrm{mL}$ penicillin and $0.08 \mathrm{mg} / \mathrm{mL}$ streptomycin) supplemented with $10 \%(\mathrm{v} / \mathrm{v})$ FBS in a relative humidified atmosphere of $5 \% \mathrm{CO}_{2}$ at $37{ }^{\circ} \mathrm{C}$. HUVEC cells were cultured in DMEM medium supplemented with $10 \%(\mathrm{v} / \mathrm{v})$ FBS and $1 \%(\mathrm{v} / \mathrm{v})$ penicillin-streptomycin in a relative humidified atmosphere of $5 \% \mathrm{CO}_{2}$ at $37{ }^{\circ} \mathrm{C}$. The controls were cells treated with an equal volume of PBS (pH 7.4). 


\subsection{Cell viability}

The cytotoxicity of free DOX, free OA, H6R6-CS-g-PNVCL, DOX@H6R6-CS-g-PNVCL and (DOX/OA)@H6R6-CS-g-PNVCL in two cell lines (SKOV3 and HUVEC) was evaluated using the MTT assay. SKOV3 cells and HUVEC cells were seeded in 96-well plates at a cell density of $1 \times 10^{4}$ cells/well and both were incubated until $80 \%$ confluent. Subsequently, free DOX, free OA, H6R6-CS-g-PNVCL, DOX@H6R6-CS-g-PNVCL and (DOX/OA)@H6R6-CS-g-PNVCL (dispersed in PBS) at the concentrations of final DOX of 0, 0.5, $1,2,5$, and $10 \mu \mathrm{g} / \mathrm{mL}$ (the concentrations of OA were set to half of the concentrations of DOX) were added into the wells and incubated for $24 \mathrm{~h}$. The cells without treatment were used as controls. MTT ( $20 \mu \mathrm{L}, 5 \mathrm{mg} / \mathrm{mL})$ was added to each well and incubated for $4 \mathrm{~h}$ followed by addition of DMSO $(180 \mu \mathrm{L})$ to dissolve formazan crystals. Finally, the absorbance at $570 \mathrm{~nm}$ was measured by a microplate reader (PowerWave XS, Bio-Tek, Winooski, VT, USA). Each experiment was performed in triplicate, and the $\mathrm{IC}_{50}$ values of each $\mathrm{DOX}$ formulation were calculated.

In order to visually assess the in vitro antitumor effect, the calcein-AM/PI double-labeling method was carried out. SKOV3 cells were seeded into a 24-well plate at the cell density of $1 \times 10^{5}$ cells/well and incubated overnight in order for the cells to adhere and grow. The original medium was discarded, then free DOX, free OA, H6R6-CS-g-PNVCL, DOX@H6R6-CS-g-PNVCL and (DOX/OA)@H6R6-CS-g-PNVCL (all dispersed in PBS) were added separately (at concentrations determined according to the $\mathrm{IC}_{50}$ values of the final DOX). The SKOV3 cells were subsequently incubated with fresh serum-free medium overnight using untreated cells as controls. Each well was washed three times with PBS (pH 7.4) and the SKOV3 cells were stained with a mixed calcein-AM and PI solution for $15 \mathrm{~min}$. The live cells (green) and dead cells (red) were distinguished under an inverted fluorescent microscope (DMi8, Leica, Germany).

The Annexin V-FITC and Propidium Iodide (PI) double-staining method was employed to determine the proportion of apoptosis. SKOV3 cells were seeded into 6-well plates at density of $1 \times 10^{6}$ cells/well and cultivated for the required time for adherence, then treated separately for $4 \mathrm{~h}$ with free DOX, free OA, H6R6-CS-g-PNVCL, DOX@H6R6-CS-g-PNVCL and 
(DOX/OA)@H6R6-CS-g-PNVCL. The SKOV3 cells were washed three times with PBS (pH 7.4), the cells were collected after digestion with $0.25 \%$ trypsin (without EDTA) and termination with McCoy's 5A incomplete medium. The cells were washed twice with PBS (pH 7.4) and then Binding Buffer $(500 \mu \mathrm{L})$ was used to re-suspend the cells at a concentration of $1 \times 10^{6}$ cells $/ \mathrm{mL}$, Annexin V-FITC $(5 \mu \mathrm{L})$ and PI $(5 \mu \mathrm{L})$ were added and the cells were incubated for 15 min at RT $\left(25^{\circ} \mathrm{C}\right)$ in the dark. All the samples were determined within $1 \mathrm{~h}$ by flow cytometry (Becton Dickinson, CA, USA).

\subsection{Cellular uptake}

In order to evaluate the intracellular uptake by the tumor cells, flow cytometry was employed. SKOV3 cells were seeded into 6-well plates at $1 \times 10^{4}$ cells/well and allowed to grow for $24 \mathrm{~h}$ and were then incubated with the control, free DOX, free OA, (DOX/OA)@CS-g-PNVCL and (DOX/OA)@H6R6-CS-g-PNVCL for 4h, respectively. The cells were washed three times with PBS (pH 7.4), harvested, and then re-suspended in PBS and then the samples were subjected to flow cytometry analysis (Becton Dickinson, CA, USA).

To examine the cell-penetrating capability of H6R6 and the properties of redox-dependent targeting the tumor cells, HUVEC cells and SKOV3 cells were visualized and imaged by confocal laser scanning microscopy (CLSM). The cells were seeded into $20 \mathrm{~mm}$ culture dishes at $1 \times 10^{4}$ cells/dish and incubated until $80 \%$ confluent and the cells were then co-cultured with various formulations including free DOX, (DOX/OA)@CS-g-PNVCL and (DOX/OA)@H6R6-CS-g-PNVCL for 2 h, respectively. Separately, (DOX/OA)@H6R6-CS-g-PNVCL was incubated with cells for $2 \mathrm{~h}$ after pre-incubation for $1 \mathrm{~h}$ with GSH (10 mM GSH) solution as a reducing reagent. All cells were washed three times with PBS (pH 7.4) and fixed for 15 min with aqueous glutaraldehyde solution $(1 \mathrm{~mL}, 2.5 \% \mathrm{v} / \mathrm{v})$. The cell nuclei were stained with Hoechst 33342 solution $(0.5 \mathrm{~mL}, 10 \mu \mathrm{g} / \mathrm{mL})$ for $15 \mathrm{~min}$, after which the cells were again washed three times with PBS (pH 7.4) and observed by CLSM (FV1000 microscope, Olympus, Japan).

\subsection{In vivo biodistribution}


Subcutaneous models of SKOV3 tumor-bearing nude mice were established by inoculating them with a suspension $\left(150 \mu \mathrm{L}, 2 \times 10^{6}\right.$ cells $)$ of SKOV3 cells in the axillary region of nude mice. The nude mice were randomized into two groups ( 8 mice each) when the tumor size reached about $0.15 \mathrm{~cm}^{3}$ in volume.

In order to track the real-time bio-distribution of fluorescence, DiR labeled nanocomposite (replacing DOX and OA with DiR), free DiR and DiR@H6R6-CS-g-PNVCL were administered by caudal vein injection with the equivalent dosage of DiR based on mouse body weight per $\mathrm{kg}$. The mice were anesthetized for real-time fluorescence imaging performed at $1 \mathrm{~h}, 4 \mathrm{~h}, 8 \mathrm{~h}$ and $24 \mathrm{~h}$ post-injection using a Maestro in vivo imaging system (CRi Inc., U.S.A.). The mice were then euthanized and the main organs (heart, liver, spleen, lung and kidney) and tumor regions were harvested for ex vivo imaging to further study the distribution of the nanocomposite.

\subsection{In vivo antitumor efficacy}

SKOV3 xenograft nude mice models were established by subcutaneous injection as described above. When the volume of the tumor grew to around $0.15 \mathrm{~cm}^{3}$, SKOV3 tumor-bearing mice were randomly assigned into six groups (eight mice each). The mice in each group were intravenously injected, every two days, with saline, free DOX, free OA, H6R6-CS-g-PNVCL, DOX@H6R6-CS-g-PNVCL and (DOX/OA)@H6R6-CS-g-PNVCL through the tail vein. The survival times of the mice were recorded and analyzed by Kaplan-Meier methodology. The dosages of various formulations were calculated as an equivalent dosage of DOX per $\mathrm{kg}$ of mouse body weight. Finally, the xenografted nude mice in the different treatment groups were weighed and the tumor sizes were measured.

\subsection{Pathological evaluation}

For further pathological study, the mice were dissected at the end of the treatment, the tumors and main organs (heart, liver, spleen, lung and kidney) in different treatment groups were collected and analyzed by hematoxylin and eosin (H\&E) staining. Furthermore, the tumor tissue sections from various treatment groups were obtained and stained using terminal deoxynucleotidyl transferase mediated dUTP nick end labeling (TUNEL) and observed with an optical microscope. 


\subsection{Statistical analysis}

Statistical analysis was carried out using either Student's t-test or one-way analysis of variance (ANOVA) combined with Tukey's post-hoc analysis (SPSS software, version 18.0, SPSS Inc.) where $p<0.05$ was considered to be significant $(*)$ and $p<0.01$ highly significant $(* *)$.

\section{Results and discussion}

\subsection{Preparation and characterization of the nanocomposites}

The synthetic route to (DOX/OA)@H6R6-CS-g-PNVCL is shown in Scheme 2. It begins with fabrication of the preliminary nanocomposite by RAFT polymerization to form the graft copolymer. H6R6 was then bound to CS-g-PNVCL with the aid of thioglycolic acid and cysteine to form the triple (redox, $\mathrm{pH}$ and temperature) sensitive nanocomposite (H6R6-CS-g-PNVCL). Finally, two anticancer agents (DOX and OA) were loaded onto the H6R6-CS-g-PNVCL to obtain (DOX/OA)@H6R6-CS-g-PNVCL having a DOX loading and encapsulation efficiency of 13.2\% and $78.6 \%$, respectively; the OA values were $7.3 \%$ and $69.9 \%$, respectively. Zeta potential are provided to demonstrate the load of the drugs in Supplementary information (Fig. S3). The (DOX/OA)@H6R6-CS-g-PNVCL NPs retain the thermosensitive properties of PNVCL, showed a phase transition at $35-42^{\circ} \mathrm{C}$ with an $\operatorname{LCST}$ of $36^{\circ} \mathrm{C}$ (Fig. 1D).

H6R6-CS-g-PNVCL was characterized by FT-IR spectroscopy, as shown in Fig. 1A and the major characteristic peaks of CS are observed at $3370 \mathrm{~cm}^{-1}\left(\mathrm{O}-\mathrm{H}\right.$ stretch); $1641 \mathrm{~cm}^{-1}$ (amide $\mathrm{C}=\mathrm{O}$ str); $1567 \mathrm{~cm}^{-1}$ (amide $\mathrm{NH}$ bend); $1629 \mathrm{~cm}^{-1}$ (C=O str of PNVCL); $1439 \mathrm{~cm}^{-1}\left(\mathrm{NVCL}-\mathrm{CH}_{2}\right)$, and $2932 \mathrm{~cm}^{-1}$ (polymeric aliphatic C-H str.). It is notable that the double bond of NVCL at 1652 $\mathrm{cm}^{-1}$ had disappeared, suggesting that the NVCL monomer had been polymerized. FT-IR spectrum of CS-RAFT and CS-g-PNVCL are provided in Supplementary information (Fig. S1). ${ }^{1} \mathrm{H}$ NMR spectra of CS-RAFT is provided in Supplementary information (Fig. S2). ${ }^{13} \mathrm{C}$ NMR spectra of $N$-CS-RAFT exhibited signals at 168.7-172.3 ppm (the carbonyl carbon as Fig. 1B), indicating the successful introduction of RAFT reagent. The TGA of CS and CS-RAFT are provided (Fig. S4), showing the successful formation of CS-RAFT and the grafting rate of RAFT 
reagent is $21.83 \%$. The above data strongly indicates the formation of CS-g-PNVCL but there was no obvious evidence that the H6R6 peptide was grafted onto the modified CS. Therefore, ${ }^{1} \mathrm{H}$ NMR spectra was used to further confirm the successful preparation of H6R6-CS-g-PNVCL (Fig. 1C). Signals at 3.1-3.8 ppm (glucosamine units H-3 to H-6, H-6' of CS); 1.9-2.6 ppm (multiple peaks $2 \mathrm{H}$ and $-\mathrm{COCH}_{2}-$ of the PNVCL units); $1.3-1.7 \mathrm{ppm}\left(-\mathrm{NH}_{2}-\mathrm{CH}_{2^{-}}\right.$of arginine in H6R6 peptide); $8.3 \mathrm{ppm}$ (imidazole ring from histidine in H6R6 peptide). All demonstrated the successful synthesis of H6R6-CS-g-PNVCL.

The representative transmission electron microscopy (TEM) image of (DOX/OA)@H6R6-CS-g-PNVCL showed that the sample presented uniform and typical spherical morphology with an average size of around $190 \mathrm{~nm}$ (Fig. 1E), corresponding to the scanning electron microscopy (SEM) results (Fig. 1F). Additionally, dynamic light scattering (DLS) measurements revealed that the average hydrodynamic diameter of the (DOX/OA)@H6R6-CS-g-PNVCL NPs was approximately 235 nm (Fig. 1G), which is slightly bigger than that in the corresponding TEM and SEM micrographs as a result of the samples having been dried before observation. Fig. $1 \mathrm{G}$ revealed that the size distribution of unloaded H6R6-CS-g-PNVCL NPs approximated $221 \mathrm{~nm}$, suggesting the successful loading of drugs for (DOX/OA)@H6R6-CS-g-PNVCL_ NPs. Importantly, the size of (DOX/OA)@H6R6-CS-g-PNVCL NP can meet the required conditions of the EPR effect.
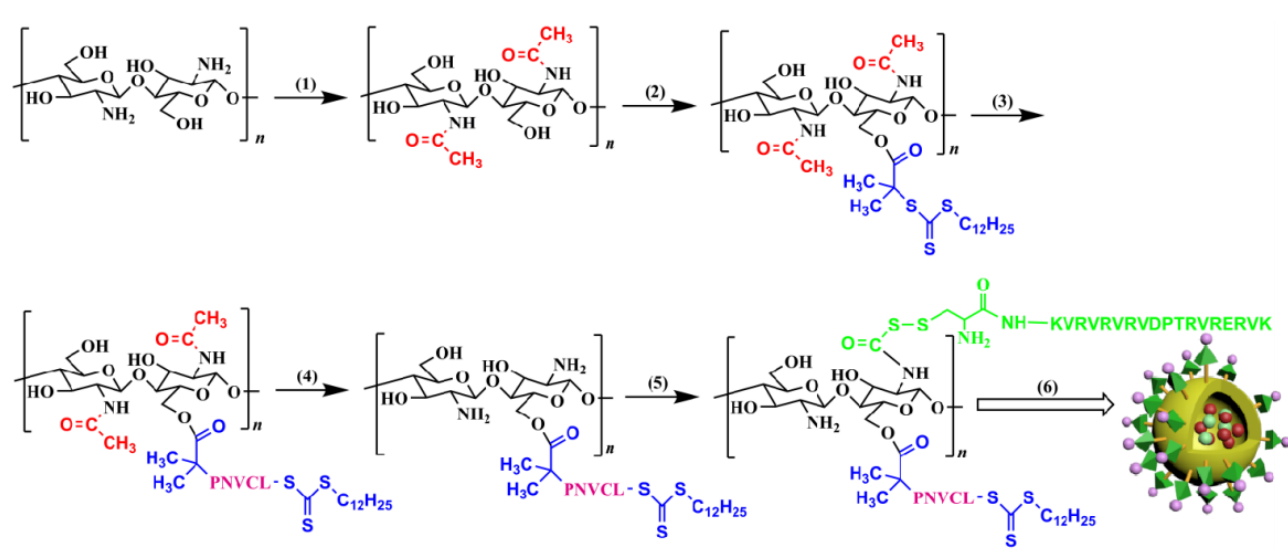

Scheme 2. The synthesis of (DOX/OA)@H6R6-CS-g-PNVCL NPs. (1) Acetic anhydride, rt, 4 h; (2) DDACT, DCC, DMAP, rt, 40 h; (3) NVCL, AIBN, $60^{\circ} \mathrm{C}, 24$ h; (4) Hydrolysis, rt; (5) Cys-H6R6, thioglycolic acid, EDC, NHS; (6) DOX, OA, self-assembly. 


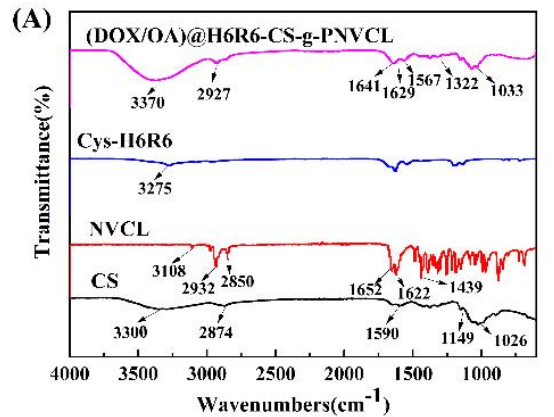

(B)
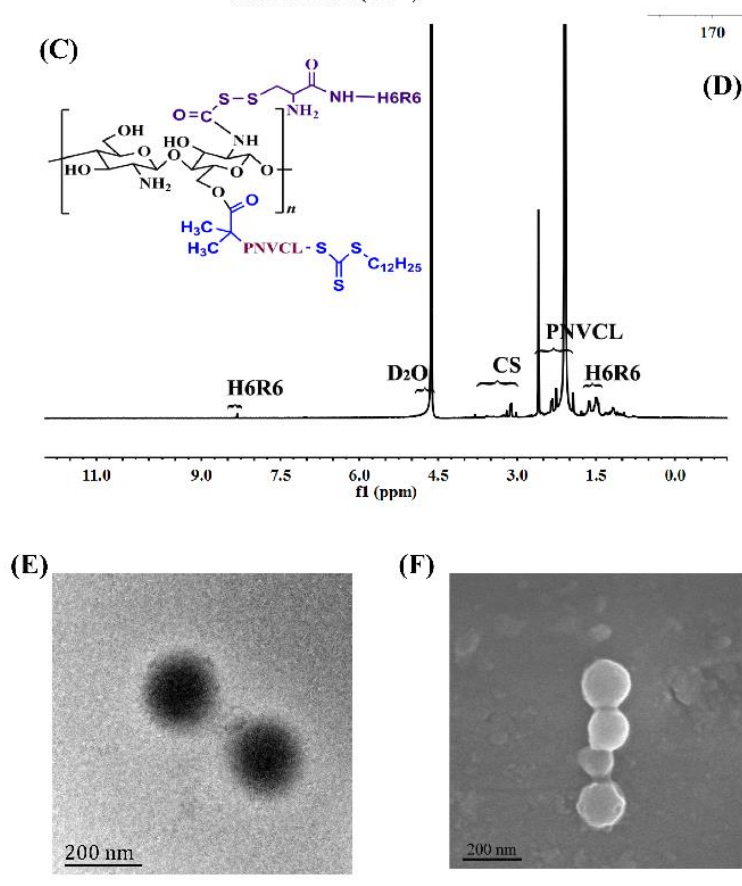

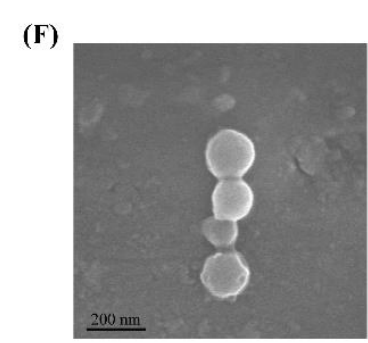

(G)

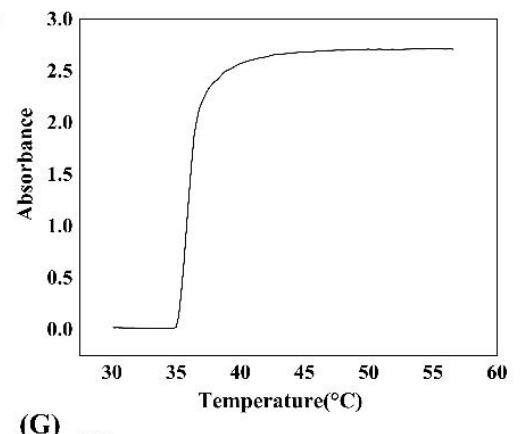

Fig. 1. Characterization of NPs: (A) FT-IR spectrum of CS, NVCL, Cys-H6R6, (DOX/OA)@H6R6-CS-g-PNVCL. (B) ${ }^{13} \mathrm{C}$ NMR spectra of $\mathrm{N}$-CS-RAFT in DMSO-d6. (C) ${ }^{1} \mathrm{H}$ NMR spectrum of (DOX/OA)@H6R6-CS-g-PNVCL in $\mathrm{D}_{2} \mathrm{O}$. (D) The change in absorbance at $500 \mathrm{~nm}$ of an aqueous solution of (DOX/OA)@H6R6-CS-g-PNVCL with temperature. (E) TEM and (F) SEM images of (DOX/OA)@H6R6-CS-g-PNVCL NPs. (G) Size distribution of H6R6-CS-g-PNVCL and (DOX/OA)@H6R6-CS-g-PNVCL NPs determined by DLS.

\subsection{Temperature/pH-triggered controlled release}

The release profiles of DOX and OA from (DOX/OA)@H6R6-CS-g-PNVCL NPs were investigated at $37^{\circ} \mathrm{C}$ and $40^{\circ} \mathrm{C}$ and $\mathrm{pH}$ values of 7.4 and 6.5. As shown in Figs. 2A and B, the DOX and OA release behaviors from (DOX/OA)@H6R6-CS-g-PNVCL NPs are affected by both temperature and $\mathrm{pH}$. The general characteristic was from rapid release to slow release of DOX and $\mathrm{OA}$ at different temperature and $\mathrm{pH}$ values within $72 \mathrm{~h}$ and the cumulative release of DOX 

$\mathrm{pH}=7.4$. The cumulative release of $\mathrm{OA}$ from the nanocomposite was $63.2 \%\left(40{ }^{\circ} \mathrm{C}, \mathrm{pH}=6.5\right)$, but only $21.1 \%$ at $37^{\circ} \mathrm{C}, \mathrm{pH}=7.4$. It is postulated that the PNVCL and $\mathrm{CS}$ from (DOX/OA)@H6R6-CS-g-PNVCL responded to the temperature and $\mathrm{pH}$ of the tumor microenvironment, resulting in the disintegration of the nanocomposite. Interestingly, a similar release trend was found for DOX and OA under similar conditions, indicating that both drugs were loaded into the nanocomposite in a similar way. These results show that the nanocomposite possesses the properties of temperature/pH-dependent triggered release for two these drugs in the tumor microenvironment.

(A)

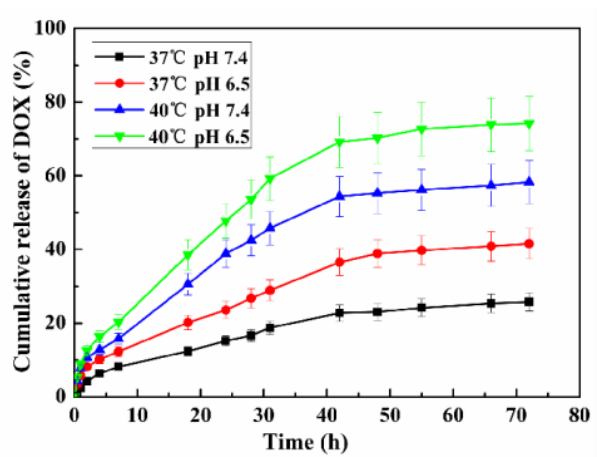

(B)

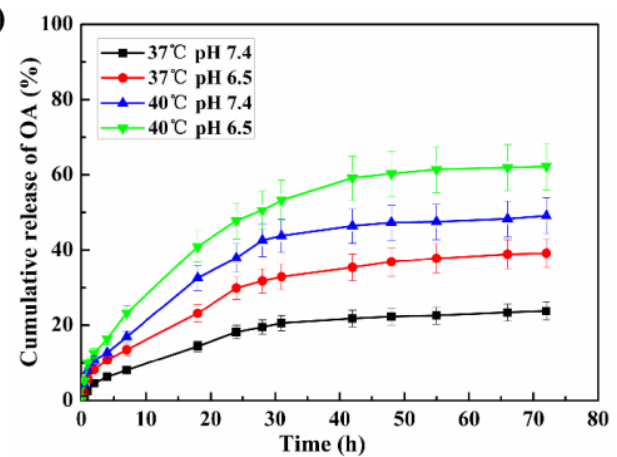

421

Fig. 2. In vitro cumulative release of (A) DOX and (B) OA from (DOX/OA)@H6R6-CS-g-PNVCL NPs at different temperatures and $\mathrm{pH}$ values. Each value is shown as mean $\pm \mathrm{SD}(n=3)$.

\subsection{Cytotoxicity studies}

Good biocompatibility is a prerequisite for successful biological applications, so in vitro cytotoxicity of various formulations on HUVEC and SKOV3 cells was studied using the MTT assay. The results suggested that blank H6R6-CS-g-PNVCL had a slight cytotoxic effect on tumor cells, but were essentially nontoxic on normal cells $(>90 \%$ cell viability). The cytotoxicity of free DOX was dose-dependent, showing more cytotoxicity toward normal cells compared to that of all other treatment groups (Fig. 3A). Meanwhile, both DOX@H6R6-CS-g-PNVCL and (DOX/OA)@H6R6-CS-g-PNVCL treatment groups exhibited much more cytotoxicity toward tumor cells than normal cells (Fig. 3A and B). However, a significant enhanced antitumor effect was achieved and attributed to the combined activity of DOX and OA (Fig. 3B). 
The in vitro antitumor efficacy was visually assessed by distinguishing between live cells (green) and dead cells (red). As shown in Fig. 3C, the DOX@H6R6-CS-g-PNVCL treatment group showed more apoptosis than the free DOX group, indicating that H6R6-CS-g-PNVCL could deliver the anti-tumor drugs to the cells more efficiently. Notably, the most apoptotic cells were observed in the (DOX/OA)@H6R6-CS-g-PNVCL treatment group compared to other groups (Fig. 3C), echoing the MTT assay results (Fig. 3B).

To further determine the antitumor effectiveness of co-delivery and mono-delivery formulations against SKOV3 cells, the sum of the rates of early and late apoptosis was calculated as the total apoptotic ratios of SKOV3 cells. As shown in Fig. 3D, the results suggested that the (DOX/OA)@H6R6-CS-g-PNVCL group had the highest total apoptosis ratio (91.3\%) compared to the controls (2.33\%), H6R6-CS-g-PNVCL (4.67\%), free OA (5.23\%), free DOX (7.75\%) and DOX@H6R6-CS-g-PNVCL (32.21\%). Additionally, the DOX@H6R6-CS-g-PNVCL treatment group showed a lower level of cell apoptosis (32.21\%) compared to the (DOX/OA)@H6R6-CS-g-PNVCL treatment group (91.3\%). These results indicate that (DOX/OA)@H6R6-CS-g-PNVCL possess the most potent tumor cell-killing capability and the antitumor efficacy of co-delivery is better than that of mono-delivery.

Two conclusions can be drawn from the above data: (1) H6R6-CS-g-PNVCL possesses good biocompatibility for normal cells and can be considered as an antitumor complement in terms of its slight cytotoxicity against tumor cells and (2)(DOX/OA)@H6R6-CS-g-PNVCL had relatively higher cytotoxicity toward tumor cells than DOX@H6R6-CS-g-PNVCL, indicating a significant enhanced antitumor activity of co-delivery in comparison to mono-delivery. 

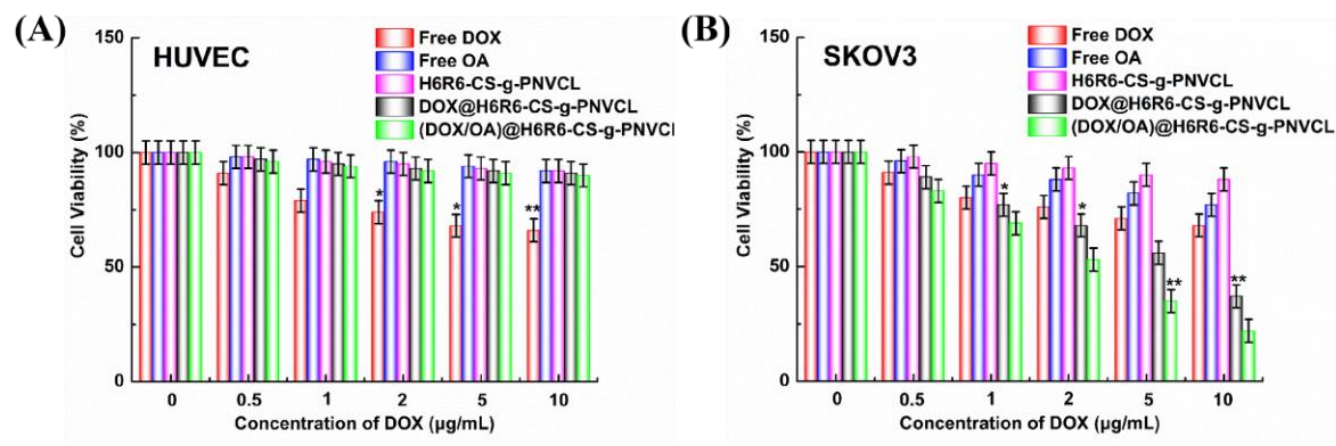

(C)
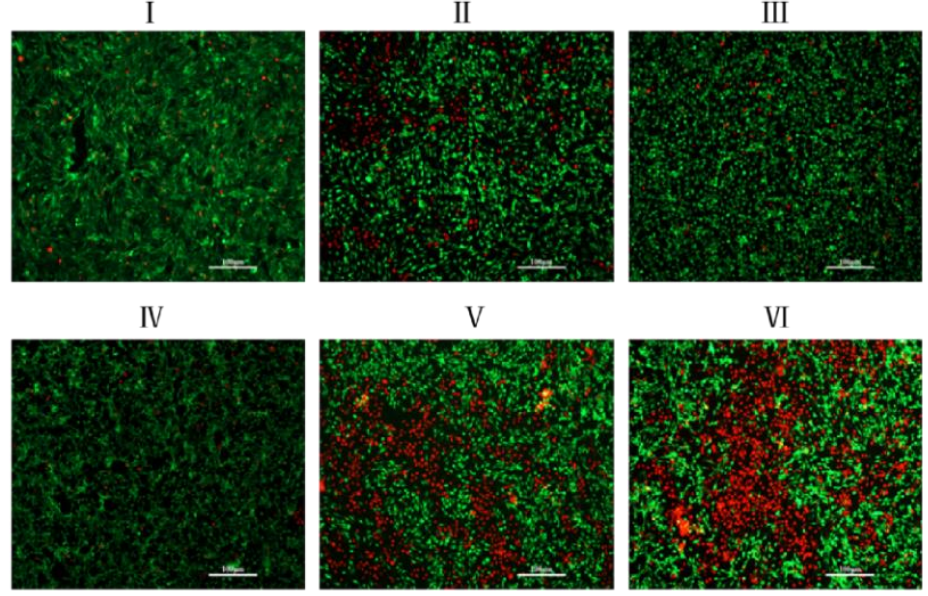

(D)

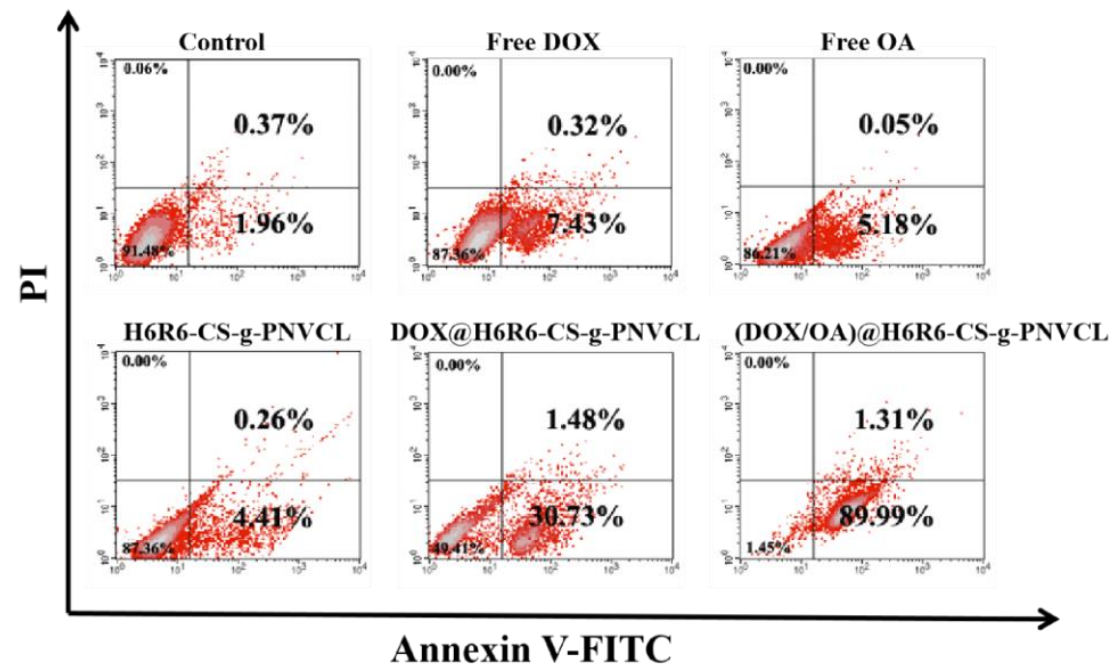

Annexin V-FITC

460 Fig. 3. Cell viability of (A) HUVEC and (B) SKOV3 cells after incubation for $24 \mathrm{~h}$ with free DOX, free OA, H6R6-CS-g-PNVCL, DOX@H6R6-CS-g-PNVCL and (DOX/OA)@H6R6-CS-g-PNVCL. Data are expressed as mean $\pm \mathrm{SD}(\mathrm{n}=3) ; * p<0.05, * * p<0.01$

(C) Live (green) /dead (red) cell assay of SKOV3 cells treated with the above various formulations: I Control; II Free DOX; III Free OA; IV H6R6-CS-g-PNVCL; V DOX@H6R6-CS-g-PNVCL; VI DOX/OA)@H6R6-CS-g-PNVCL. (scale bars =100 $\mu \mathrm{m}$ ).

(D) Flow cytometry analysis of SKOV3 cell apoptosis induced by free DOX, free OA, H6R6-CS-g-PNVCL, assay. 


\subsection{Reduction-responsive controlled intracellular uptake}

470

471

472

473

474

475

In order to evaluate the internalization of tumor cells for various treatment groups, intracellular uptake was measured by flow cytometry and CLSM. According to the results of flow cytometry, the uptake curve of the (DOX/OA)@H6R6-CS-g-PNVCL group shifted more to the right than that of the control, free DOX, free OA and (DOX/OA)@CS-g-PNVCL groups, revealing that the H6R6-functionalized NPs were taken up by SKOV3 cells due to the superior penetrating function of the H6R6 peptide (Fig. 4A).

CLSM results showed that red fluorescence signals of HUVEC cells incubated with (DOX/OA)@CS-g-PNVCL and (DOX/OA)@H6R6-CS-g-PNVCL were quite weak. However, SKOV3 cells incubated with (DOX/OA)@H6R6-CS-g-PNVCL exhibited a much higher red fluorescence signal compared to those treated with (DOX/OA)@CS-g-PNVCL, indicating that H6R6-functionalized NPs could target tumor cells, rather than normal cells (Fig. 4B). Using H6R6-functionalized NPs pretreated with GSH to culture cells, the red fluorescence signals of HUVEC cells and SKOV3 cells were almost invisible (Fig. 4B) because when H6R6-functionalized NPs reach normal cells, they rarely stay there due to the cell-penetrating function of H6R6 peptide. In contrast, once H6R6-functionalized NPs reach tumor cells, the H6R6 peptide is released via breakage of the -S-S- bond under the reductive tumor microenvironment, causing NPs to stay in the tumor cells. These results indicate the cell-penetrating capability of the H6R6 peptide and the function of reduction-responsive controlled targeting of tumor cells by the nanocomposite. 
(A)
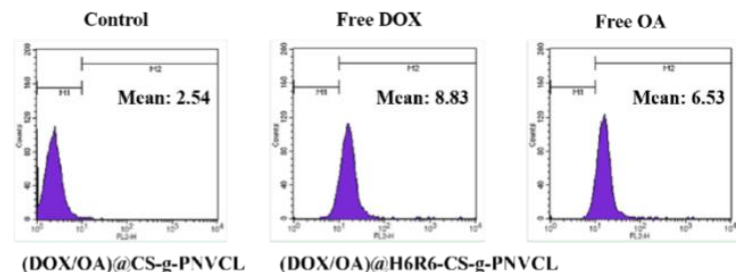

(DOX/OA) (a) H6R6-CS-g-PNVCL
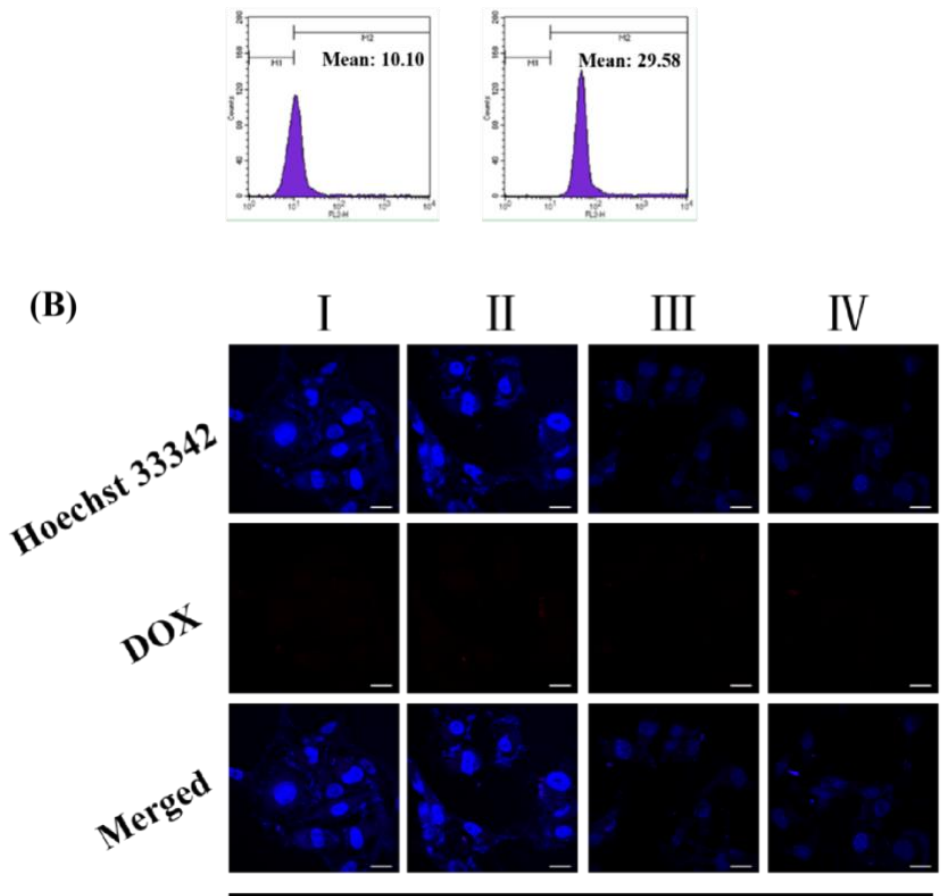

HUVEC

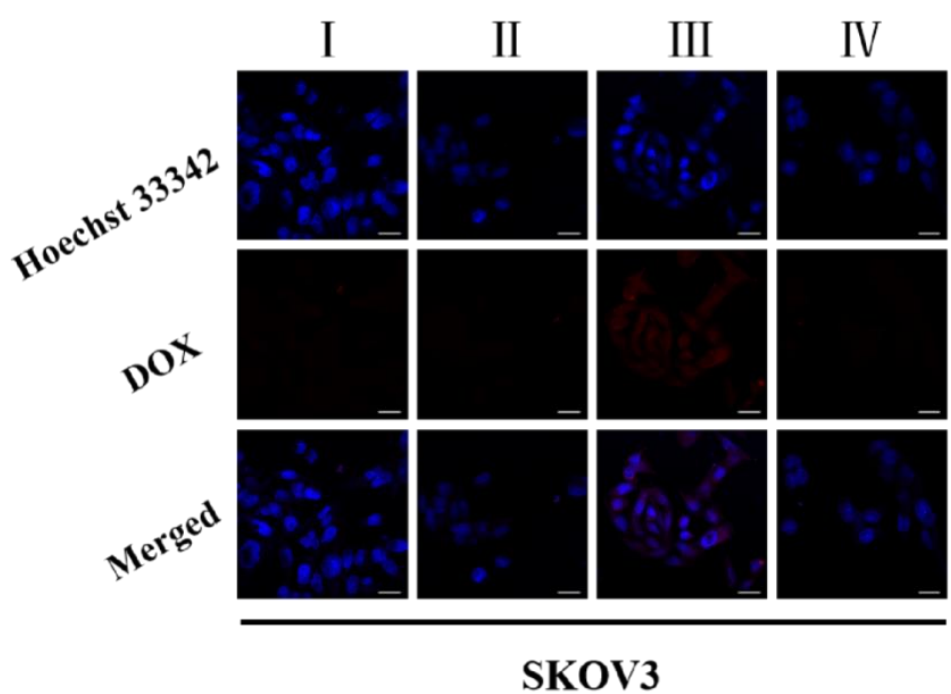

491 Fig. 4. Cell uptake studies. (A) Flow cytometry analysis of internalization of free DOX, free OA, 492 (DOX/OA)@CS-g-PNVCL and (DOX/OA)@H6R6-CS-g-PNVCL on SKOV3 cells. (B) CLSM images of 493 HUVEC cells and SKOV3 cells after incubation with ( I ) free DOX; ( II) (DOX/OA)@CS-g-PNVCL; (III) 494 (DOX/OA)@H6R6-CS-g-PNVCL; and (IV) (DOX/OA)@H6R6-CS-g-PNVCL with GSH (scale bar = $50 \mu \mathrm{m})$. 


\subsection{In vivo biodistribution}

497

In order to study in vivo biodistribution of the H6R6-functionalized NPs, fluorescence DiR

499 labeled NPs were observed. As shown in Fig. 5A, the DiR labeled NPs group presented strong

500 fluorescence at the tumor site in the first $1 \mathrm{~h}$, whereas the fluorescence of free DiR group was not

501 observed at the tumor site within the same period. All fluorescence decreased over time as a

502 result of metabolism, but some distinct fluorescence could still be seen even $24 \mathrm{~h}$ after injection.

503 After $24 \mathrm{~h}$, the free DiR group showed accumulation in other areas in addition to the tumor region

504 whilst the DiR labeled NPs group displayed a higher accumulation in the tumor region.

505 Additionally, ex vivo imaging results showed distributions of free DiR and DiR labeled NPs in

506 major organs and tumors, in which free DiR existed in major healthy organs as well as the tumor

507 areas as a result of non-specific biodistribution but DiR labeled NPs mainly accumulated in tumor

508 areas with little liver deposition (Fig. 5B). According to the quantitative region of interest (ROI)

509 analysis, the accumulation in the tumor of DiR labeled NPs was 2.25-fold higher than that of free

510 DiR (Fig. 5C). These results demonstrate that H6R6-functionalized NPs are feasible for in vivo

511 use and possess a favorable EPR effect with fewer side effects. 


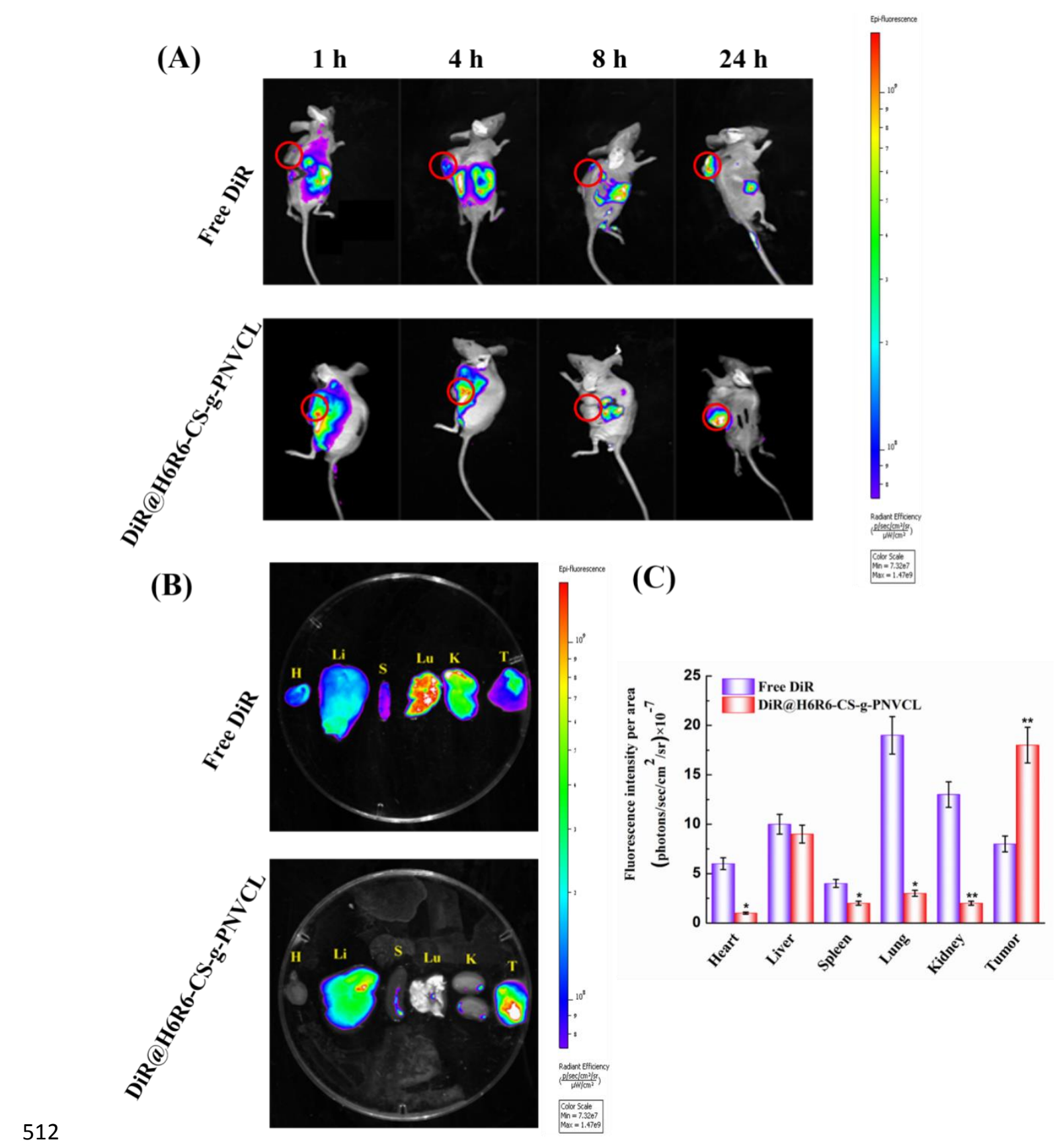

513 Fig. 5. (A) In vivo fluorescence images of SKOV3 xenograft nude mice after i.v. injection of free DiR and

514 DiR@H6R6-CS-g-PNVCL NPs (images taken from different mice). The red circles indicate the tumor sites.

(B)

515 Ex vivo fluorescence images and (C) statistical analysis of hearts (H), livers (Li), spleens (S), lungs (Lu), kidneys

$516(\mathrm{~K})$ and tumors $(\mathrm{T}) . \quad$ Data are given as mean $\pm \mathrm{SD}(n=8) . \quad * p<0.05, * * p<0.01$.

\subsection{In vivo therapeutic efficacy evaluation}

Encouraged by the above results, the in vivo antitumor effect of various treatments were

521 studied. The saline group showed rapid tumor growth over the $30 \mathrm{~d}$, while almost all of the other 
treatments inhibited tumor growth (Fig. 6A) due to the presence of CS from blank H6R6-CS-g-PNVCL hiving some anti-tumor effects. The tumor growth inhibition using (DOX/OA)@H6R6-CS-g-PNVCL and DOX@H6R6-CS-g-PNVCL treatment groups were found to be the best and co-delivery, in comparison to mono-delivery, achieved a significantly enhanced antitumor efficacy (Fig. 6A). Additionally, the body weights of the mice from various treatment groups showed no obvious differences, except for the free DOX treatment group which was probably due to high systemic toxicity (Fig. 6B). Fig. 6C showed the mean survival duration of the mice in the various groups with saline being 43 days, the H6R6-CS-g-PNVCL group 44 days, the free OA group 49 days and the free DOX group 38 days. In contrast, the mice treated with (DOX/OA)@H6R6-CS-g-PNVCL and DOX@H6R6-CS-g-PNVCL survived for over 60 days with survival rates of around $64 \%$ and $42 \%$, respectively. These results thereby verify the outstanding in vivo antitumor effect of (DOX/OA)@H6R6-CS-g-PNVCL NPs, suggesting that the co-delivery system has a better performance on inhibiting tumor growth than that of mono-delivery.

(A)

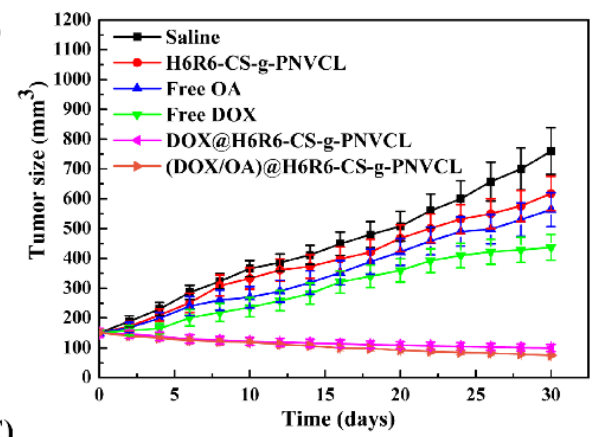

(C)

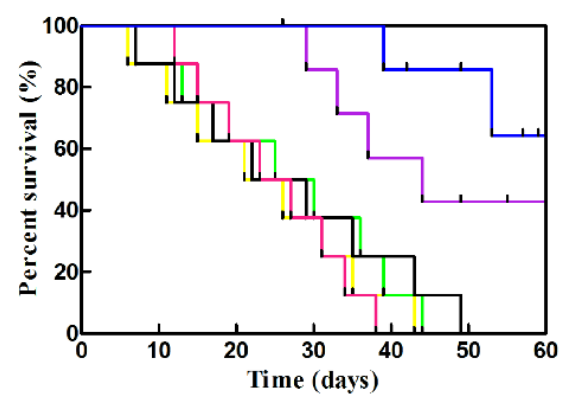

(B)

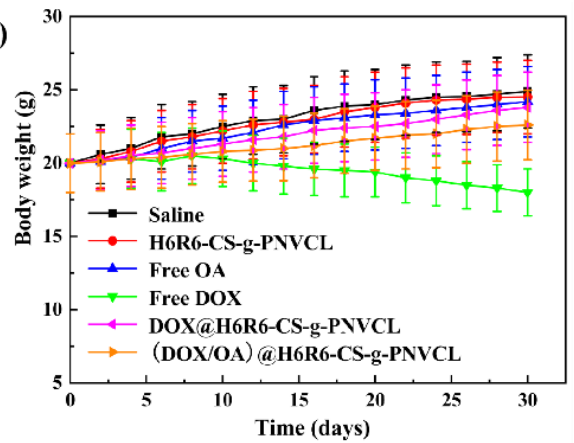

- Saline

ᄂ H6R6-CS-g-PNVCL

- Free $\mathrm{OA}$

+ Free Dox

十 DOX@H6R6-CS-g-PNVCL

-(DOX/OA)@H6R6-CS-g-PNVCL

Fig. 6. In vivo antitumor effects of different treatments.

(A) Tumor growth curves of the mice treated with different formulations.

(B) Body weights of mice in different treatment groups as indicated over 30 days.

(C)

Kaplan-Meier survival curves $(n=8)$. 


\subsection{Pathological evaluation}

The H\&E staining images (Fig. 7A) indicated that the (DOX/OA)@H6R6-CS-g-PNVCL treatment group exhibited the highest detrimental effect on tumor tissue, while there was no evidence of damage in the main organs, implying low systemic toxicity. Except for the free DOX group, no significant pathological variations were observed in the main organs for the remaining treatment groups. However, free OA and DOX@H6R6-CS-g-PNVCL groups displayed some abnormal tissue morphologies in tumors, meaning that $\mathrm{OA}$ and DOX@H6R6-CS-g-PNVCL were also somewhat toxic to tumors but not normal organs.

TUNEL staining images were used for further evaluation of the toxicity in tumor tissue in the different treatment groups. As displayed in Fig. 7B, normal cell nuclei were stained dark blue while the apoptotic ones were brown. In line with the anti-tumor results in vivo, the largest areas of apoptosis were observed in the (DOX/OA)@H6R6-CS-g-PNVCL treatment group. Furthermore, the group of mono-delivery of DOX also showed many apoptotic cells, but fewer than that shown by co-delivery of DOX and OA. Quantitative analyses of the TUNEL staining images (Fig. 7C) showed the apoptosis rates of tumor cells are 7\% for saline, $12 \%$ for H6R6-CS-g-PNVCL, 20\% for free OA, 46\% for free DOX, 63\% for DOX@H6R6-CS-g-PNVCL, and $86 \%$ for the (DOX/OA)@H6R6-CS-g-PNVCL treatment group. Overall, these results fully demonstrate the rationality of the design of this triple sensitive nanocomposite and the superiority of the two anticancer agents in synergy. 


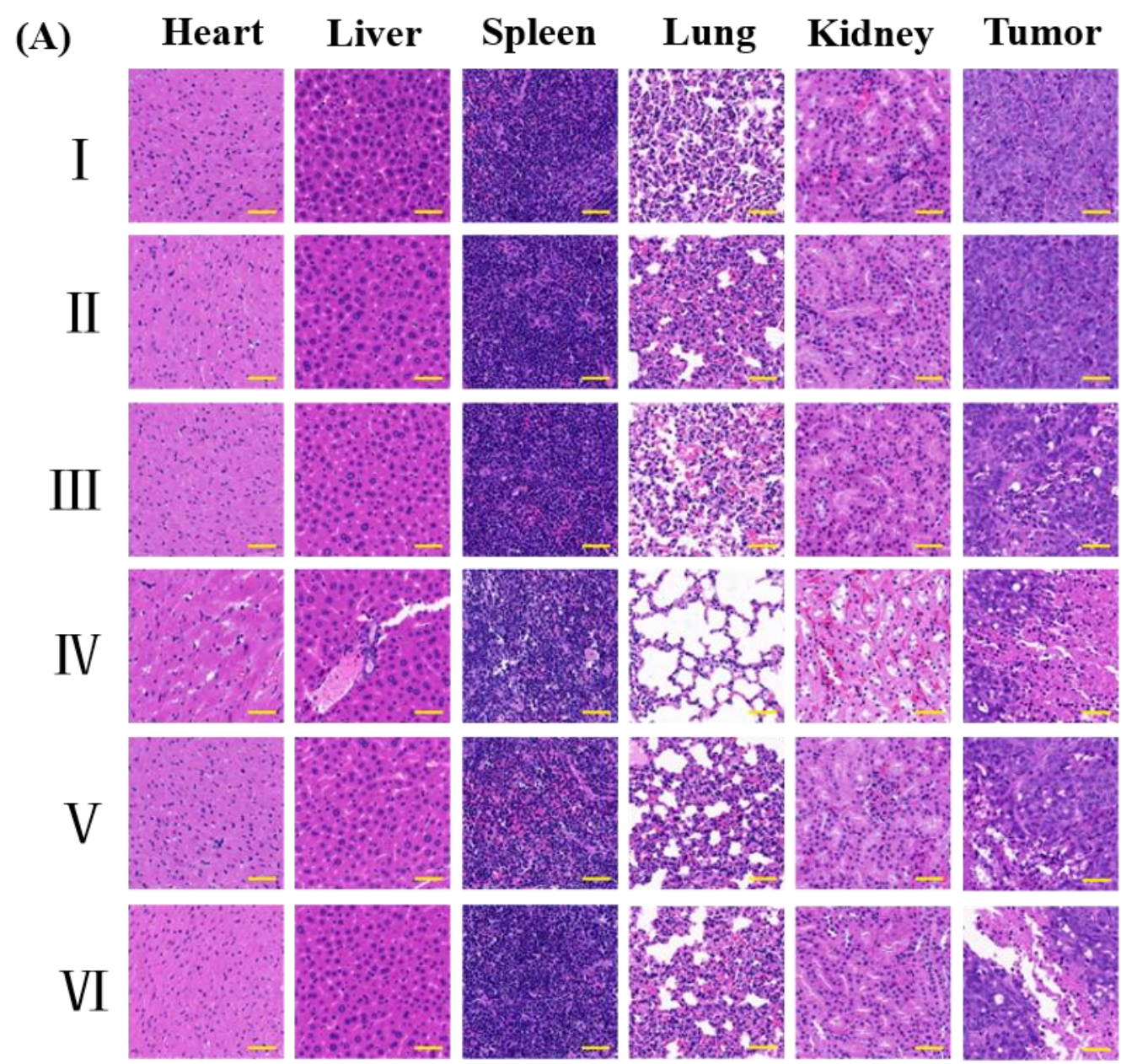

(B)

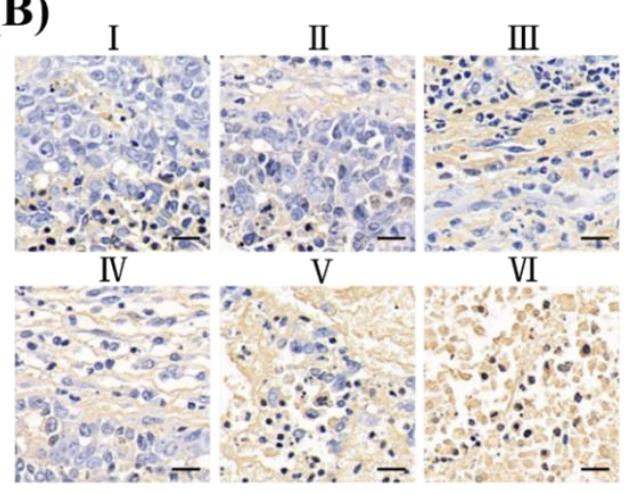

(C)

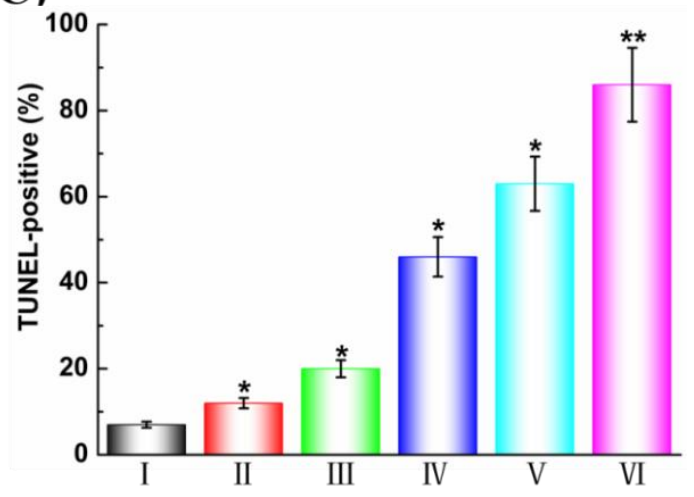

Fig. 7. Pathological analyses. (A) Histological analysis of the main organ and tumor slices from various

567 Statistical analysis of the TUNEL positive rates. ( I Saline; II H6R6-CS-g-PNVCL; III Free OA; IV Free DOX;

568 V DOX@H6R6-CS-g-PNVCL; VI (DOX/OA)@H6R6-CS-g-PNVCL). Data are shown as mean \pm SD $(n=5)$.

$* p<0.05, * * p<0.01$ 


\section{Conclusion}

In summary, the development of a triply sensitive nanocomposite that possesses the ability of selective residence in tumor cells due to presence of the H6R6 peptide and reduction-responsive disulfide bonds (-S-S-) is described. Remarkable is the influence of $\mathrm{pH}$ or temperature on DOX and OA release profiles from nanocomposite based on chitosan. The release characteristics of DOX and OA are from rapid release to slow release, then reaching a plateau at different temperature and $\mathrm{pH}$. The cumulative release of $\mathrm{DOX}$ or $\mathrm{OA}$ is higher at acidic $\mathrm{pH}(6.5)$ and higher temperature $\left(40^{\circ} \mathrm{C}\right)$ than at physiological $\mathrm{pH}(7.4)$ and temperature $\left(37^{\circ} \mathrm{C}\right)$. The release of DOX is maximum (about 74.6\%) at specific $\mathrm{pH}$ and temperature of simulated tumor microenvironment $\left(\mathrm{pH}=6.5,40^{\circ} \mathrm{C}\right.$ ), while DOX release is minimal (about $24.7 \%$ ) at physiological $\mathrm{pH}$ and temperature $\left(\mathrm{pH}=7.4,37^{\circ} \mathrm{C}\right)$. The cumulative release of $\mathrm{OA}$ from the nanocomposite is $63.2 \%\left(\mathrm{pH}=6.5,40{ }^{\circ} \mathrm{C}\right)$, but only $21.1 \%\left(\mathrm{pH}=7.4,37^{\circ} \mathrm{C}\right)$. The system demonstrates the property of triggering DOX and OA release as a result of temperature/pH dual-responsive decomposition behavior. The co-delivery system demonstrates fully the advantage of tumor microenvironment characteristics to achieve targeted and controlled release. Both in vitro and in vivo studies show that the co-delivery system exhibits greater chemotherapeutic effects compared to monotherapy and such a co-delivery strategy shows great promise for enhanced development in cancer chemotherapy. This triple sensitive co-delivery system could be applied in treatment of a variety of solid tumor types to overcome many difficulties of conventional chemotherapy.

\section{Disclosure statement}

The authors declare no conflict of interest.

\section{Acknowledgments}

This research was financially supported by grant 16410723700 from the Science and Technology Commission of Shanghai Municipality, the Biomedical Textile Materials "111 Project" of the Ministry of Education of China (No. B07024), the UK China Joint Laboratory for Therapeutic Textiles (based at Donghua University) and the National Natural Science Foundation 
of China (81460647).

\section{References}

Chai, Z., Ran, D., Lu, L., Zhan, C., Ruan, H., Hu, X., . . L Lu, W. (2019). Ligand-Modified Cell Membrane Enables the Targeted Delivery of Drug Nanocrystals to Glioma. ACS Nano, 13(5), 5591-5601.

Chen, W.-H., Luo, G.-F., Qiu, W.-X., Lei, Q., Liu, L.-H., Zheng, D.-W., . . . Zhang, X.-Z. (2016). Tumor-Triggered Drug Release with Tumor-Targeted Accumulation and Elevated Drug Retention To Overcome Multidrug Resistance. Chemistry of Materials, 28(18), 6742-6752.

Chen, X., Niu, T., Gao, Y., Liang, X., Li, S., Zhang, L., . . . Wang, C. (2019). Tunable synthesis of $\mathrm{pH}$-responsive biodegradable $\mathrm{ZnO}$ nanospheres assembled from ultrasmall particles for cancer chemotherapy. Chemical Engineering Journal, 371, 443-451.

Chen, Z., Huang, K. Y., Ling, Y., Goto, M., Duan, H. Q., Tong, X. H., . . Lee, K. H. (2019). Discovery of an Oleanolic Acid/Hederagenin-Nitric Oxide Donor Hybrid as an EGFR Tyrosine Kinase Inhibitor for Non-Small-Cell Lung Cancer. J Nat Prod, 82(11), 3065-3073.

El-Sawy, H. S., Al-Abd, A. M., Ahmed, T. A., El-Say, K. M., \& Torchilin, V. P. (2018). Stimuli-Responsive Nano-Architecture Drug-Delivery Systems to Solid Tumor Micromilieu: Past, Present, and Future Perspectives. ACS Nano, 12(11), 10636-10664.

Galbiati, A., Tabolacci, C., Morozzo Della Rocca, B., Mattioli, P., Beninati, S., Paradossi, G., \& Desideri, A. (2011). Targeting tumor cells through chitosan-folate modified microcapsules loaded with camptothecin. Bioconjug Chem, 22(6), 1066-1072.

Gao, G., Jiang, Y. W., Jia, H. R., Sun, W., Guo, Y., Yu, X. W., . . Wu, F. G. (2019). From perinuclear to intranuclear localization: A cell-penetrating peptide modification strategy to modulate cancer cell migration under mild laser irradiation and improve photothermal therapeutic performance. Biomaterials, 223, 119443.

Hingorani, D. V., Chapelin, F., Stares, E., Adams, S. R., Okada, H., \& Ahrens, E. T. (2020). Cell penetrating peptide functionalized perfluorocarbon nanoemulsions for targeted cell labeling and enhanced fluorine-19 MRI detection. Magn Reson Med, 83(3), 974-987.

Hu, H., Li, Y., Zhou, Q., Ao, Y., Yu, C., Wan, Y., . . . Yang, X. (2016). Redox-Sensitive Hydroxyethyl Starch-Doxorubicin Conjugate for Tumor Targeted Drug Delivery. ACS Appl Mater Interfaces, 8(45), 30833-30844.

Indulekha, S., Arunkumar, P., Bahadur, D., \& Srivastava, R. (2017). Dual responsive magnetic composite nanogels for thermo-chemotherapy. Colloids Surf B Biointerfaces, 155, 304-313.

Jin, Z., Nguyen, K. T., Go, G., Kang, B., Min, H. K., Kim, S. J., . . . Choi, E. (2019). Multifunctional Nanorobot System for Active Therapeutic Delivery and Synergistic Chemo-photothermal Therapy. Nano Lett.

Ke, W., Li, J., Mohammed, F., Wang, Y., Tou, K., Liu, X., . . . Ge, Z. (2019). Therapeutic Polymersome Nanoreactors with Tumor-Specific Activable Cascade Reactions for Cooperative Cancer Therapy. ACS Nano, 13(2), 2357-2369.

Lang, J., Zhao, X., Qi, Y., Zhang, Y., Han, X., Ding, Y., . . . Nie, G. (2019). Reshaping Prostate Tumor Microenvironment To Suppress Metastasis via Cancer-Associated Fibroblast Inactivation with Peptide-Assembly-Based Nanosystem. ACS Nano, 13(11), 12357-12371. 
Liu, X., Liu, J., Liu, D., Han, Y., Xu, H., Liu, L., . . Kong, D. (2019). A cell-penetrating peptide-assisted nanovaccine promotes antigen cross-presentation and anti-tumor immune response. Biomater Sci, 7(12), 5516-5527.

Luo, W., Xu, X., Zhou, B., He, P., Li, Y., \& Liu, C. (2019). Formation of enzymatic/redox-switching nanogates on mesoporous silica nanoparticles for anticancer drug delivery. Mater Sci Eng C Mater Biol Appl, 100, 855-861.

Mao, H., Xie, Y., Ju, H., Mao, H., Zhao, L., Wang, Z., . . . Liu, H. (2018). Design of Tumor Microenvironment-Responsive Drug-Drug Micelle for Cancer Radiochemotherapy. ACS Appl Mater Interfaces, 10(40), 33923-33935.

Montes, J. Á., Ortega, A., \& Burillo, G. (2014). Dual-stimuli responsive copolymers based on $\mathrm{N}$-vinylcaprolactam/chitosan. Journal of Radioanalytical and Nuclear Chemistry.

Niu, S., Bremner, D. H., Wu, J., Wu, J., Wang, H., Li, H., . . . Zhu, L. (2018). I-Peptide functionalized dual-responsive nanoparticles for controlled paclitaxel release and enhanced apoptosis in breast cancer cells. Drug Deliv, 25(1), 1275-1288.

Niu, S., Williams, G. R., Wu, J., Wu, J., Zhang, X., Chen, X., . . . Zhu, L.-M. (2019). A chitosan-based cascade-responsive drug delivery system for triple-negative breast cancer therapy. Journal of Nanobiotechnology, 17(1).

Niu, S., Williams, G. R., Wu, J., Wu, J., Zhang, X., Zheng, H., . . . Zhu, L.-M. (2019). A novel chitosan-based nanomedicine for multi-drug resistant breast cancer therapy. Chemical Engineering Journal, 369, 134-149.

Perche. (2019). Stimuli-Sensitive Cell Penetrating Peptide-Modified Nanocarriers. Processes, 7(10).

Prabaharan, M., Grailer, J. J., Steeber, D. A., \& Gong, S. (2009). Thermosensitive micelles based on folate-conjugated poly(N-vinylcaprolactam)-block-poly(ethylene glycol) for tumor-targeted drug delivery. Macromol Biosci, 9(8), 744-753.

Qian, Q., Niu, S., Williams, G. R., Wu, J., Zhang, X., \& Zhu, L.-M. (2019). Peptide functionalized dual-responsive chitosan nanoparticles for controlled drug delivery to breast cancer cells. Colloids and Surfaces A: Physicochemical and Engineering Aspects, 564, 122-130.

Quinones, J. P., Peniche, H., \& Peniche, C. (2018). Chitosan Based Self-Assembled Nanoparticles in Drug Delivery. Polymers (Basel), 10(3).

Rosiere, R., Van Woensel, M., Gelbcke, M., Mathieu, V., Hecq, J., Mathivet, T., . . Wauthoz, N. (2018). New Folate-Grafted Chitosan Derivative To Improve Delivery of Paclitaxel-Loaded Solid Lipid Nanoparticles for Lung Tumor Therapy by Inhalation. Mol Pharm, 15(3), 899-910.

Silva, A. M., Alvarado, H. L., Abrego, G., Martins-Gomes, C., Garduno-Ramirez, M. L., Garcia, M. L., . . . Souto, E. B. (2019). In Vitro Cytotoxicity of Oleanolic/Ursolic Acids-Loaded in PLGA Nanoparticles in Different Cell Lines. Pharmaceutics, 11(8).

Sun, L., Jiang, W., Zhang, H., Guo, Y., Chen, W., Jin, Y., . . . Wang, B. (2019). Photosensitizer-Loaded Multifunctional Chitosan Nanoparticles for Simultaneous in Situ Imaging, Highly Efficient Bacterial Biofilm Eradication, and Tumor Ablation. ACS Appl Mater Interfaces, 11(2), 2302-2316.

Sun, P., Huang, W., Kang, L., Jin, M., Fan, B., Jin, H., . . . Gao, Z. (2017). siRNA-loaded poly(histidine-arginine)6-modified chitosan nanoparticle with enhanced cell-penetrating and endosomal escape capacities for suppressing breast tumor metastasis. Int J Nanomedicine, 12, 3221-3234.

Takemura, M., Endo, S., Matsunaga, T., Soda, M., Zhao, H. T., El-Kabbani, O., . . Hara, A. (2011). 
Selective inhibition of the tumor marker aldo-keto reductase family member $1 \mathrm{~B} 10$ by oleanolic acid. J Nat Prod, 74(5), 1201-1206.

Tao, R., Gao, M., Liu, F., Guo, X., Fan, A., Ding, D., . . Zhao, Y. (2018). Alleviating the Liver Toxicity of Chemotherapy via pH-Responsive Hepatoprotective Prodrug Micelles. ACS Appl Mater Interfaces, 10(26), 21836-21846.

Wang, J., Wang, X., Lu, S. Y., Hu, J., Zhang, W., Xu, L., . . Liu, H. (2019). Integration of cascade delivery and tumor hypoxia modulating capacities in core-releasable satellite nanovehicles to enhance tumor chemotherapy. Biomaterials, 223, 119465.

Wang, S., Zhang, S., Liu, J., Liu, Z., Su, L., Wang, H., \& Chang, J. (2014). pH- and reduction-responsive polymeric lipid vesicles for enhanced tumor cellular internalization and triggered drug release. ACS Appl Mater Interfaces, 6(13), 10706-10713.

Wu, H., Zhuang, Q., Xu, J., Xu, L., Zhao, Y., Wang, C., . . . Peng, R. (2019). Cell-Penetrating Peptide Enhanced Antigen Presentation for Cancer Immunotherapy. Bioconjug Chem, 30(8), 2115-2126.

Xiao, J., Zhang, G., Xu, R., Chen, H., Wang, H., Tian, G., . . . Wu, Z. (2019). A pH-responsive platform combining chemodynamic therapy with limotherapy for simultaneous bioimaging and synergistic cancer therapy. Biomaterials, 216, 119254.

Xiao, L., Huang, L., Moingeon, F., Gauthier, M., \& Yang, G. (2017). pH-Responsive Poly(Ethylene Glycol)-block-Polylactide Micelles for Tumor-Targeted Drug Delivery. Biomacromolecules, 18(9), 2711-2722.

Xu, J., Khan, A. R., Fu, M., Wang, R., Ji, J., \& Zhai, G. (2019). Cell-penetrating peptide: a means of breaking through the physiological barriers of different tissues and organs. J Control Release, 309, 106-124.

Yang, G., Chen, C., Zhu, Y., Liu, Z., Xue, Y., Zhong, S., . . . Zhang, W. (2019). GSH-Activatable NIR Nanoplatform with Mitochondria Targeting for Enhancing Tumor-Specific Therapy. ACS Appl Mater Interfaces.

Zhang, X., Niu, S., Williams, G. R., Wu, J., Chen, X., Zheng, H., \& Zhu, L. M. (2019). Dual-responsive nanoparticles based on chitosan for enhanced breast cancer therapy. Carbohydr Polym, 221, 84-93.

Zhang, Y., Dang, M., Tian, Y., Zhu, Y., Liu, W., Tian, W., . . . Lu, G. (2017). Tumor Acidic Microenvironment Targeted Drug Delivery Based on pHLIP-Modified Mesoporous Organosilica Nanoparticles. ACS Appl Mater Interfaces, 9(36), 30543-30552.

Zhang, Y., Lu, Y., Zhang, Y., He, X., Chen, Q., Liu, L., . . Jiang, C. (2017). Tumor-Targeting Micelles Based on Linear-Dendritic PEG-PTX8 Conjugate for Triple Negative Breast Cancer Therapy. Mol Pharm, 14(10), 3409-3421.

Zhao, C., Shao, L., Lu, J., Deng, X., Tong, Y., \& Wu, Y. (2017). Hybrid Prodrug Nanoparticles with Tumor Penetration and Programmed Drug Activation for Enhanced Chemoresistant Cancer Therapy. ACS Appl Mater Interfaces, 9(22), 18450-18461.

Zhou, L., Lv, F., Liu, L., Shen, G., Yan, X., Bazan, G. C., \& Wang, S. (2018). Cross-Linking of Thiolated Paclitaxel-Oligo(p-phenylene vinylene) Conjugates Aggregates inside Tumor Cells Leads to "Chemical Locks" That Increase Drug Efficacy. Adv Mater, 30(10). 